\title{
Relación entre Inteligencia Emocional, Satisfacción Vital, Felicidad Subjetiva y Resiliencia en funcionarios de Educación Especial
}

\author{
Relationship between Emotional Intelligence with Life Satisfaction, Subjective Happi- \\ ness and Resilience in Special Education employees
Relação entre Inteligência Emocional com Satisfação Vital, Felicidade Subjetiva e Resiliência em funcionários da Educação Especial

\section{Constanza Veloso-Besio, a Alejandro Cuadra-Peralta, Ivonne Antezana-Saguez, Rosa Avendaño-Robledo, Leslie Fuentes-Soto}

${ }^{a}$ Universidad de Tarapacá, Departamento de Psicología, Velázquez 2222, Arica, Chile.

Correo electrónico: veloso.constanza@gmail.com

\begin{abstract}
RESUMEN
El propósito de este estudio fue analizar la relación de inteligencia emocional percibida con satisfacción vital, felicidad subjetiva y resiliencia, en una muestra de 117 funcionarios de educación especial. Para esto se utilizaron los siguientes instrumentos: Trait Meta-Mood Scale y Emotional Quotient Inventory, ambos para medir inteligencia emocional; Escala de Satisfacción Vital; Escala de Felicidad Subjetiva y para medir resiliencia se utilizó la Connor-Davidson Resilience Scale. Los resultados evidenciaron que inteligencia emocional percibida se relacionó con las variables mencionadas, destacándose la correlación obtenida con resiliencia.
\end{abstract}

Palabras clave: inteligencia emocional, educación especial, regresión múltiple.

\begin{abstract}
The purpose of this study was to analyze the relationship between perceived emotional intelligence with life satisfaction, subjective happiness and resilience in a sample of special education employees who work in special education. Data were collected by using a Trait Meta-Mood Scale and an Emotional Quotient Inventory, both to measure emotional intelligence, Life Satisfaction Scale, Subjective Happiness Scale, and the Connor-Davidson Resilience Scale. The results showed that perceived emotional intelligence was related to the previously mentioned variables, highlighting the correlation obtained with resilience.
\end{abstract}

Key words: emotional intelligence, special education, multiple regression.

\section{RESUMO}

Objetiva-se analisar a relação de inteligência emocional percebida com satisfação vital, felicidade subjetiva e resiliência em uma amostra de 117 funcionários de educação especial. Para isso, foram utilizados os seguintes instrumentos: o Trait Meta-Mood Scale e o Emotional Quotient Inventory, ambos com finalidade de medir a inteligência emocional; Escala de Satisfacción Vital, Escala de Felicidad Subjetiva e, para medir a resiliência, foi utilizada a Connor-Davidson Resilience Scale. Os resultados evidenciaram que a inteligência emocional percebida relacionou-se com as variáveis mencionadas, destacando-se a correlação obtida com resiliência.

Palavras chave: inteligência emocional, educação especial, regressão múltiplas. 


\section{RELACIÓN ENTRE INTELIGENCIA EMOCIONAL CON SATISFACCIÓN VITAL, FELICIDAD SUBJETIVA Y RESILIENCIA, EN FUNCIONARIOS DE EDUCACIÓN ESPECIAL}

Se espera que el trabajo brinde la posibilidad de formarse como persona y, a la vez, suponer una fuente de satisfacciones. Estas satisfacciones se producen en la medida en que la actividad a la cual el sujeto se dedica resulte agradable, ofrezca el reconocimiento social de los demás, aporte recursos económicos que se consideran suficientes, etc. Sin embargo, en el ámbito educativo, específicamente en la educación especial, no siempre la práctica laboral aporta dichas satisfacciones a los trabajadores (Cuadra-Peralta, LeeMaturana, Sosa- Roa, 2009; Paula, 2005). Es más, la educación especial, en la provisión de servicios para personas con discapacidad, constituye un colectivo especialmente afectado por factores de riesgo que este trabajo comporta (Schneider y Bowen, 1993), como alteraciones del sistema nervioso, especialmente neurosis y otras enfermedades psiquiátricas (Caballero y Valero, 2004).

A la hora de explicar por qué son estas profesiones las más afectadas, autores como Maslach y Jackson (1986) señalan que a los profesionales asistenciales se les pide que se impliquen intensamente con personas que, por lo general, se encuentran en una situación problemática en la que tienen cabida sentimientos como la frustración, el miedo y la desesperación, por lo que la tensión resultante puede tener un efecto de desgaste emocional. Así, se puede decir que las emociones juegan un papel primordial en este tema, y que la capacidad para razonar sobre ellas, percibirlas y comprenderlas permite desarrollar procesos de regulación emocional que ayudarían a moderar y prevenir los efectos negativos del estrés (Extremera, Durán y Rey, 2010; Extremera, Fernández-Berrocal y Durán, 2003).

Los antecedentes planteados anteriormente dan cuenta de la importancia de la inteligencia emocional en la percepción de la actividad que llevan a cabo estos trabajadores y en su grado de bienestar personal y laboral. De este modo, se señala la posibilidad de mejorar la calidad de vida laboral a través del fomento de sus habilidades emocionales, favoreciendo el desarrollo de habilidades prácticas y de manejo del estrés, un mayor bienestar percibido y la mejora de la calidad de los servicios ofrecidos a los usuarios (Extremera, Durán y Rey, 2005).

Por tanto, resulta de interés examinar la relación entre el nivel de inteligencia emocional y el bienestar personal, laboral y psicológico de colectivos tales como el de trabajadores que atienden a personas con discapacidad intelectual, pues la tensión inherente de su actividad y el constante contacto con usuarios y familiares pueden favorecer el deterioro de la satisfacción y afectar negativamente al desempeño de su labor (Gil-Monte y Peiró, 1996).

\subsection{INTELIGENCIA EMOCIONAL}

El concepto de inteligencia emocional fue acuñado el año 1990 por Peter Salovey y John Mayer, quienes concibieron la inteligencia emocional como una inteligencia genuina, basada en el uso adaptativo de las emociones, de manera que el individuo pudiese solucionar problemas y adaptarse de forma eficaz al medio que le rodea (FernándezBerrocal y Ruiz, 2008).

Al introducir el concepto a la literatura científica, y en el intento de aportar una medida que sirviera como referencia de la inteligencia emocional, fueron desarrollados 
diferentes modelos del constructo, los cuales se pueden clasificar en dos tipos: modelos de habilidades y modelos mixtos (Mayer, Roberts y Barsade, 2008).

Los modelos de habilidades se centran en la capacidad para percibir, comprender y manejar la información que proporcionan las emociones (Mayer, Salovey y Caruso, 2000). Categoría en que se destaca el modelo de Mayer y Salovey, el cual conceptualiza la inteligencia emocional a través de cuatro habilidades básicas: percepción emocional, facilitación emocional, comprensión emocional y regulación emocional (Cabello, RuizAranda y Fernández-Berrocal, 2009). Respecto de la medición del constructo una de las medidas de autoinforme más utilizadas es la Trait Meta-Mood Scale (TMMS, Salovey, Mayer, Goldman, Turvey y Palfai, 1995), la cual se compone de tres factores: atención, claridad emocional y reparación de emociones.

Los modelos mixtos combinan habilidades mentales con características de personalidad (Mayer et al., 2008), categoría en que se encuentra el modelo de Bar-On (1997), quien define la inteligencia emocional como un conjunto de capacidades emocionales, personales e interpersonales que influyen en la capacidad total del individuo para hacer frente a las demandas y a las presiones del medio ambiente (Ugarriza, 2001). Este modelo distingue los siguientes factores: habilidades intrapersonales, habilidades interpersonales, adaptabilidad, manejo de estrés y estado anímico general.

Es menester mencionar que dichos modelos de inteligencia emocional parten de dos bases distintas de análisis, por un lado, los modelos de habilidades se centran en las emociones y sus interacciones con el pensamiento, mientras que los modelos mixtos alternan las habilidades mentales con una variedad de otras características (Mayer et al., 2000). No obstante, los modelos mencionados de inteligencia emocional comparten un núcleo común de conceptos básicos, verbigracia, a nivel general, la inteligencia emocional se refiere a las habilidades para reconocer y regular emociones en nosotros mismos y en los otros. Asimismo, dichos modelos tienen como noción de base la insuficiencia del intelecto para operar de forma óptima sin la inteligencia emocional. De esta manera, se busca el balance entre el coeficiente intelectual y la lógica con la emoción y nuestras capacidades personales, emocionales y sociales (Gabel, 2005).

\subsection{BIENESTAR SUBJETIVO: SATISFACCIÓN VITAL Y FELICIDAD SUBJETIVA}

El bienestar subjetivo incluye dos componentes claramente diferenciados y que han seguido líneas de investigación paralelas: por un lado, los juicios cognitivos sobre satisfacción con la vida y, por otro, las evaluaciones afectivas sobre el humor y las emociones, equivalentes a la felicidad subjetiva (Padrós, Martínez, Gutiérrez-Hernández y Medina, 2010). El componente cognitivo hace alusión al constructo de satisfacción vital, el cual se define como la valoración positiva que la persona hace de su vida en general, o de aspectos particulares de ésta, tales como: familia, estudios, trabajo, salud, amigos, tiempo libre (Diener, Suh, Lucas y Smith, 1999; García, 2000; Liberalesso, 2002), siendo los dominios de funcionamiento más cercanos e inmediatos a la vida personal de los individuos los que tendrían mayor influencia sobre el bienestar personal.

Distintos investigadores han puesto en evidencia la relación entre los constructos de inteligencia emocional y satisfacción vital, entre los que se encuentran Palomera y Brackett (2000), quienes hallaron que la satisfacción vital correlacionó significativamente con claridad emocional $(r=, 29)$ y reparación emocional $(r=, 25)$. Extremera, Durán y Rey (2005) 
también reportaron correlaciones estadísticamente significativas entre satisfacción vital con claridad emocional $(r=, 32)$ y reparación emocional $(r=, 25)$. Páez, Fernández, Campos, Zubieta y Casullo (2006) informaron que el índice de déficit en inteligencia emocional se vinculó negativamente con la satisfacción con la vida en general $(r=-, 20)$. Incluso se ha visto que al utilizar programas basados en componentes de inteligencia emocional se puede modificar el nivel de inteligencia emocional (Cuadra, Fuentes, Madueños, Bustos y Veloso, 2011; Cuadra-Peralta, Veloso-Besio, Ibergaray y Rocha, 2010; Cuadra-Peralta, Veloso-Besio, Moya-Rubio, Reyes y Vilca, 2010; Cuadra-Peralta; Veloso-Besio, PudduGallardo, Salgado-García, en prensa).

El componente emocional, alusivo a la felicidad subjetiva, corresponde a la preponderancia de los sentimientos o afectos positivos sobre los negativos, donde el afecto positivo puede ser evaluado a través de emociones específicas como gozo, afecto y orgullo; en tanto que el afecto negativo puede ser medido por medio de emociones o sentimientos tales como vergüenza, culpa, tristeza, ira o ansiedad. Una persona que tenga experiencias emocionales positivas, es más probable que perciba su vida satisfactoriamente. Así mismo, los sujetos que valoran positivamente su vida tendrán más emociones positivas que negativas (Zamarrón, 2006). La definición de bienestar, desde esta perspectiva, está más cercana al uso cotidiano que se hace de este término. En este sentido, se entiende que una persona es más feliz cuando en su vida predomina la experiencia afectiva positiva sobre la negativa (García, 2002).

Fernández-Berrocal y Extremera (2009) señalan que desde que Mayer y Salovey, en 1990, incorporaran el constructo de inteligencia emocional en la literatura científica, diversos estudiosos han investigado el vínculo de las habilidades emocionales sobre el bienestar individual y la felicidad subjetiva. Por ejemplo, Extremera et al. (2005) encontraron correlaciones positivas y estadísticamente significativas entre felicidad subjetiva con claridad emocional $(r=, 34)$ y reparación $(r=, 39)$.

De acuerdo con García (2002) existe evidencia empírica que las dimensiones cognitiva y afectiva del bienestar subjetivo están altamente relacionadas. Sin embargo, una de las principales dificultades para llevar a cabo esta verificación es la carencia de medidas adecuadas, ya que muchas de las escalas de satisfacción vital a menudo contienen componentes afectivos. A pesar de esto, la mayor parte de los investigadores encuentran útil dicha distinción en la comprensión del concepto de bienestar subjetivo.

\subsection{RESILIENCIA}

Seligman y Czikszentmihalyi (2000), autores pertenecientes a la corriente de la psicología positiva, sostienen que el estudio de la resiliencia debería centrarse fundamentalmente en las fortalezas y aspectos positivos presentes en los seres humanos. Es decir, más que centrarse en las condiciones de alto riesgo para la salud física y mental de las personas, debería centrarse en estudiar las condiciones que posibilitan un desarrollo más sano y positivo. Se trata de superar el modelo de enfermedad por una perspectiva que promueva y valore las potencialidades, capacidades, talentos y recursos propios. Así, la resiliencia contribuye a la calidad de vida, además de ser un amortiguador y facilitador para enfrentar con éxito el estrés.

Salovey, Rothman, Detweiler y Steward (2000) mencionan los efectos que tienen las experiencias emocionales positivas, en cuanto a que proveen mejores recursos para 
poder prevenir enfermedades o afrontar más efectivamente los problemas de salud ya existentes. Los autores afirman que los estados emocionales positivos facilitan conductas más saludables, proveyéndoles la resiliencia necesaria para afrontar la posibilidad de tener o desarrollar serios problemas de salud.

Cabe señalar que la resiliencia se encuentra dentro los temas que han comenzado a ser abordados por diversos investigadores interesados en los aspectos positivos del ser humano (Hervás, 2009). Sin embargo, según Bonanno (2004), la mayoría de las investigaciones en resiliencia se han centrado en la población infantil y adolescente, por lo que resulta de interés estudiar cómo se manifiesta la resiliencia en los adultos, que se desempeña en el área de la educación especial, y qué relación presenta con inteligencia emocional.

En consideración a los antecedentes expuestos, las hipótesis de trabajo son:

Hipótesis 1: Inteligencia emocional correlaciona positivamente con satisfacción vital.

Hipótesis 2: Inteligencia emocional correlaciona positivamente con felicidad subjetiva.

Hipótesis 3: Inteligencia emocional correlaciona positivamente con resiliencia.

\section{MÉTODO}

Participantes: Ciento diecisiete funcionarios de establecimientos de educación especial de las ciudades de Arica e Iquique. De éstos, $17,9 \%(\mathrm{~N}=21)$ eran hombres y 82,1\% $(\mathrm{N}=96)$ eran mujeres. El 55,6\% $(\mathrm{N}=65)$ trabajan en establecimientos municipalizados y $44,4 \%(\mathrm{~N}=52)$ trabajan en establecimientos particulares-subvencionados. La técnica de muestreo empleada fue de carácter no probabilístico, por disponibilidad.

Variables: Inteligencia emocional, definida como el puntaje obtenido por los sujetos en dos escalas diferentes: Trait Meta-Mood Scale-24 y Emocional Quotient Inventory; satisfacción vital; felicidad subjetiva y resiliencia.

Instrumentos: Para medir inteligencia emocional, se utilizaron dos medidas de evaluación. En primer lugar, se utilizó la adaptación española de Fernández-Berrocal, Extremera y Ramos (2004) de la Trait Meta-Mood Scale-24 (TMMS-24, Salovey et al., 1995). Este instrumento consta de 24 ítems en la que los sujetos evalúan su grado de acuerdo sobre una escala tipo Likert de cinco opciones (1= mиy de acuerdo; 5=mиy en desacuerdo). El cuestionario está compuesto por tres factores: atención emocional, implica el grado en que las personas creen prestar atención a sus sentimientos; claridad emocional, se refiere a cómo creen percibir sus emociones las personas; y reparación emocional, hace referencia a la creencia del sujeto en su capacidad para interrumpir estados emocionales negativos y prolongar los positivos.

De los tres factores de la Trait Meta-Mood Scale-24, se decidió eliminar el factor atención emocional debido a que evidenció una correlación débil con las otras dimensiones de la misma escala, con claridad la correlación fue de ,29** y con reparación de ,35**. Valores bajo lo esperado al considerar que los factores atención, claridad y reparación, forman parte de un mismo constructo. A lo anterior hay que agregar que la confiabilidad de dicha escala fue menor que 0,7 , lo cual está bajo el rango sugerido por la comunidad científica.

Una vez reducida la escala, la cual quedó formada solo por las dimensiones claridad y reparación emocional, se pudo observar que aumentaron las correlaciones entre la escala general TMMS-24 y los otros constructos (satisfacción vital, felicidad subjetiva y 
resiliencia). El coeficiente de fiabilidad alcanzado por la nueva escala TMMS-24 fue de ,88 con validez de constructo unidimensional, que explicó $37 \%$ de la varianza.

En segundo lugar, se utilizó Emotional Quotient Inventory (EQ-I) de Bar-On (2000), validado en una muestra local por Espinoza y Zúñiga (2003). Bar-On plantea varios modelos posibles para interpretar su instrumento. El más conocido, usa 15 subescalas que se agrupan en cinco subfactores (Bar-On, 1997). Sin embargo, este modelo no fue confirmado en la validación realizada en Chile por Espinoza y Zúñiga (2003). Otra manera de interpretar dicho instrumento, es considerar la inteligencia emocional constituida por 10 subescalas y cinco subescalas de apoyo (Ugarriza, 2001). Este último es confirmado en la validación chilena y consta de 65 ítems, donde cada reactivo es evaluado a través de una escala de respuesta tipo Likert de cinco opciones (1=rara vez o nunca es mi caso $; 5=$ con mucha frecuencia es mi caso). Su fiabilidad general, estimada mediante el indicador Alfa de Cronbach fue de ,82.

Para medir satisfacción vital, se utilizó la adaptación española de Atienza, Pons, Balaguer y García-Merita (2000) de la Escala de Satisfacción con la Vida de Diener, Emmons, Larsen y Griffin (1985). Esta escala es una medida global acerca del grado percibido de satisfacción en la vida. Consta de cinco ítems compuestos por una escala tipo Likert de siete alternativas ( $1=$ completamente en desacuerdo $;=$ completamente de acuerdo). Su fiabilidad general, estimada mediante el indicador Alfa de Cronbach fue de ,76. Además, evidenció validez de constructo unidimensional, con un factor que explicó $53 \%$ de la varianza.

Para medir felicidad subjetiva, se utilizó la Escala de Felicidad Subjetiva de Lyubomirsky y Lepper (1999). Consiste en una escala tipo Likert de cuatro ítems con siete alternativas de respuesta $(1=$ nada en absoluto; $7=$ en gran medida $)$. Esta escala mide la felicidad subjetiva global mediante enunciados en los que la persona se evalúa a sí mismo o bien se compara con quienes le rodean. Su fiabilidad general, estimada mediante el indicador Alfa de Cronbach fue de ,69. Además, presentó validez de constructo unidimensional, con un factor que explicó 56\% de la varianza.

Para medir resiliencia, se utilizó la Escala de Resiliencia Connor-Davidson de Connor y Davidson (2003). Cuestionario auto aplicado que evalúa cómo el participante se ha sentido en el último mes, consta de 25 ítems tipo Likert de cuatro opciones $(0=$ nada de acuerdo hasta $4=$ totalmente de acuerdo). En relación a las características psicométricas, en Estados Unidos Connor y Davidson (2003) definieron la estructura del constructo a partir de cinco factores y su consistencia interna fue ,89. En un intento por verificar estos resultados Yu y Zhang (2007), en una muestra de la población China, encontraron una estructura de tres factores y una consistencia interna de, 91 . Ante estas discrepancias psicométricas encontradas en la literatura, se decidió realizar un análisis factorial que defina la estructura del constructo. Se extrajeron los reactivos 8, 9, 11 y 22. Posteriormente, se obtuvo la validez de constructo, con un factor que explicó $30 \%$ de la varianza. La fiabilidad general estimada mediante indicador Alfa de Cronbach fue de ,87.

Diseño y Procedimiento: Se utilizó un diseño ex - post facto retrospectivo de grupo único. Para la recolección de datos se aplicaron de modo directo los instrumentos, proporcionando a cada participante un cuadernillo que contenía los seis instrumentos mencionados, enfatizando la voluntariedad de acceder a contestar y la confidencialidad de los datos a través del anonimato. El tiempo promedio de respuesta fue de 60 minutos, tarea realizada durante la jornada laboral. 


\section{RESULTADOS}

En relación a la primera hipótesis planteada (Inteligencia emocional correlaciona positivamente con satisfacción vital), el análisis de los datos reveló una correlación positiva y significativa con ambas medidas de la inteligencia emocional, para IE.TMMS-24 la correlación fue de ,48 y para IE EQ-i la correlación fue de ,45.

Respecto de la segunda hipótesis (Inteligencia emocional correlaciona positivamente con felicidad subjetiva) se tiene que la correlación entre las variables fue positiva y significativa con ambas medidas de la inteligencia emocional, para IE.TMMS-24 la correlación fue de ,58 y para IE EQ-i fue de ,57.

En relación a la tercera hipótesis planteada (Inteligencia emocional correlaciona positivamente con resiliencia) el análisis de los datos reveló una correlación positiva y significativa con ambas medidas de la inteligencia emocional, para IE.TMMS-24 la correlación obtenida fue de ,69 y para IE EQ-i fue de ,59.

Tabla 1. Correlaciones entre inteligencia emocional con satisfacción vital, felicidad subjetiva y resiliencia

\begin{tabular}{|lccc|}
\hline & $\begin{array}{c}\text { Satisfacción } \\
\text { Vital }\end{array}$ & $\begin{array}{c}\text { Felicidad } \\
\text { Subjetiva }\end{array}$ & Resiliencia \\
\hline${\text { IE TMMS-24 } \text { parcial }^{(a)}}^{(4)}$ &, $481(* *)$ &, $582(* *)$ &, $698(* *)$ \\
IE EQ-i $^{(b)}$ &, $454(* *)$ &, $571(* *)$ &, $598(* *)$ \\
\hline
\end{tabular}

4. ** Correlación significativa al .01 (bilateral).

5. (a) Inteligencia emocional medida por TMMS-24, considerando solo las dimensiones claridad y reparación.

Nombre exclusivo creado para este estudio con el objetivo de facilitar la interpretación de los datos.

6. (b) Inteligencia emocional medida por EQ-i.

Para profundizar mejor los resultados, se realizó un análisis de regresión múltiple utilizando como variable independiente las subescalas de las respectivas medidas de inteligencia emocional, TMMS-24: claridad y reparación; EQ-i: solución de problemas, tolerancia al estrés, comprensión, prueba de realidad, autoconcepto, control de impulsos, flexibilidad, relaciones interpersonales, asertividad y empatía, considerando como variables dependientes satisfacción vital, felicidad subjetiva y resiliencia. Además, se utilizó el método de extracción por pasos sucesivos (step wise) para obtener solo las variables que permitan el mejor ajuste posible.

Tabla 2. Análisis de regresión múltiple para la explicación de satisfacción vital, felicidad subjetiva y resiliencia, utilizando inteligencia emocional como variable independiente

\begin{tabular}{|lccccc|}
\hline & $\% \mathrm{R}^{2}$ & $\mathrm{~F}$ & $\mathrm{P}$ & $\mathrm{B}$ & $\mathrm{P}$ \\
\hline VD Satisfacción vital & 32,2 & 27,12 &, 000 & & \\
1.Claridad & & & &, 353 &, 000 \\
2.Autoconcepto & & & &, 310 &, 001 \\
\hline
\end{tabular}




\begin{tabular}{|c|c|c|c|c|c|}
\hline $\begin{array}{l}\text { VD Felicidad Subjetiva } \\
\text { 1.Claridad } \\
\text { 2.Autoconcepto }\end{array}$ & 39,4 & 37,08 &, 000 & $\begin{array}{l}, 377 \\
, 356\end{array}$ & $\begin{array}{l}, 000 \\
, 000\end{array}$ \\
\hline VD Resiliencia & 59,5 & 55,35 & ,000 & & \\
\hline $\begin{array}{l}\text { 1.Reparación } \\
\text { 2.Relaciones interpersonales } \\
\text { 3.Solución de problemas }\end{array}$ & & & & $\begin{array}{l}, 394 \\
, 296 \\
, 298\end{array}$ & $\begin{array}{l}, 000 \\
, 000 \\
, 000\end{array}$ \\
\hline
\end{tabular}

Con respecto a la variable dependiente satisfacción vital se tiene que solo las dimensiones de claridad $(\bigotimes=, 353 ; p=, 000)$ y autoconcepto $(\bigotimes=, 310 ; p=, 001)$ presentaron coeficientes de regresión significativos. Ambas dimensiones explicaron el 32,2\% de la variabilidad total de satisfacción vital. El modelo estadístico para la variable dependiente felicidad subjetiva quedó conformado por las dimensiones de claridad $(\mathbb{\nabla}=, 377$;

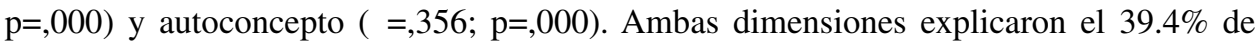
la varianza de felicidad subjetiva. Finalmente, las dimensiones de IE que contribuyeron significativamente en la explicación de resiliencia fueron: reparación $(\mathbb{Q}=, 395 ; \mathrm{p}=, 000)$, solución de problemas $(\bigotimes=, 298 ; \mathrm{p}=, 000)$ y relaciones interpersonales $(\bigotimes=, 296 ; \mathrm{p}=, 000)$. Estas dimensiones en conjunto explicaron el $59,5 \%$ de la varianza total de resiliencia.

\section{DISCUSIONES Y CONCLUSIONES}

El objetivo inicial del presente estudio era contrastar las relaciones existentes entre inteligencia emocional con satisfacción vital, felicidad subjetiva y resiliencia, en trabajadores de educación especial en las ciudades de Arica e Iquique. Cada una de estas relaciones recibió apoyo empírico. Por tanto, se puede establecer que inteligencia emocional, con ambas medidas utilizadas, se relaciona positiva y significativamente con felicidad subjetiva, satisfacción vital y resiliencia. Resultados que vienen a corroborar lo ya planteado por diferentes autores.

En relación a la primera hipótesis de trabajo, los resultados encontrados evidenciaron que inteligencia emocional correlacionó significativamente con satisfacción vital. Vale decir, en la medida que docentes y funcionaros se perciban como emocionalmente inteligentes se sentirán más satisfechos con sus vidas y vice-versa. El análisis de regresión múltiple proporciona mayor información al respecto, ya que las correlaciones directas consideraron el constructo general de inteligencia emocional (factor de segundo orden). Así se tuvo que las únicas dimensiones que explicaron un porcentaje significativo de la varianza de satisfacción vital (32\%) fueron claridad (TMMS) y autoconcepto (EQI). Estos hallazgos van en dirección similar a los informados por Palomera y Brackett (2000), Extremera et al. (2005) y Páez et al. (2006), no obstante, es menester recordar que nuestro estudio incorporó un modelo de habilidad y uno mixto en la medición de inteligencia emocional.

Respecto de la segunda hipótesis de trabajo, los resultados de la presente investigación establecen una relación positiva y significativa entre inteligencia emocional percibida y felicidad subjetiva. Esto quiere decir que una persona que tenga experiencias emocionales positivas, es más probable que perciba su vida satisfactoriamente. Así mismo, los 
sujetos que valoran positivamente su vida tendrán más emociones positivas que negativas (Zamarrón, 2006). Nuevamente es necesario mencionar los resultados obtenidos en análisis de regresión múltiple ya que proporcionan evidencia más detallada sobre cuáles son las dimensiones de inteligencia emocional que más varianza explican de la felicidad subjetiva. Solo las dimensiones de claridad (TMMS) y autoconcepto (EQI) fueron las que lograron explicar un porcentaje significativo de la varianza total de felicidad subjetiva. Además, ambas dimensiones en conjunto explicaron un 39,9\% de los niveles de felicidad subjetiva. Estos resultados convergen, en parte, con los reportados en el estudio de Extremera et al. (2005), en que claridad también dio cuenta de un porcentaje significativo de la varianza de felicidad subjetiva.

Es importante mencionar que tanto la satisfacción vital como la felicidad subjetiva son explicadas por las mismas dimensiones de la inteligencia emocional, a saber: claridad emocional y autoconcepto. Este resultado puede deberse en parte a lo señalado por García (2002), quien señala que ambos constructos están altamente relacionados.

La tercera hipótesis de trabajo también recibió apoyo empírico a favor, en términos que inteligencia emocional evidenció una correlación positiva con resiliencia. Cabe destacar que ésta variable fue la que presentó las correlaciones más altas con ambas medidas de inteligencia emocional percibida. Por tanto, es de esperar que aquellas personas que presentan la habilidad para percibir, asimilar y manejar las emociones propias y detectar e interpretar las emociones de los demás, presenten un mayor nivel de resiliencia. El análisis de regresión múltiple realizado evidenció que fueron las dimensiones de reparación (TMMS), relaciones interpersonales (EQI) y solución de problemas (EQI) las que explicaron un porcentaje significativo de la varianza de resiliencia, en conjunto dieron cuenta de un $59,5 \%$ de la variabilidad.

Dentro de las limitaciones del estudio se tiene que a pesar que la muestra estuvo conformada por cinco establecimientos educacionales, estos sólo representan las dos primeras regiones del país, reducción geográfica que se explica por el costo económico que implica incluir otras zonas que se encuentran más distantes.

A la luz de los resultados obtenidos, se sugiere continuar analizando los niveles de inteligencia emocional percibida y su relación con la resiliencia, para confirmar y clarificar el tipo de relación entre los constructos, robusteciendo el cuerpo científico al respecto.

Dado que las características de la muestra limitan en alguna medida las conclusiones a otros contextos educativos especiales, se recomienda incluir mayor heterogeneidad en cuanto a género, nivel de estudios y contextos socioeconómicos, a modo de favorecer la generalización de los resultados.

Las implicaciones prácticas de este estudio, resultan relevantes para la salud mental de los trabajadores que prestan servicios en establecimientos de educación especial. En concreto, las habilidades de inteligencia emocional ejercen efectos beneficiosos a nivel preventivo. Es decir, la capacidad para razonar sobre las emociones, percibirlas y comprenderlas, como habilidad intrínseca del ser humano, implica, en último término, el desarrollo de procesos de regulación emocional que ayudarían a prevenir, moderar y afrontar los contratiempos cotidianos y el estrés laboral al que están expuestos diariamente. Además, el cuerpo de funcionarios debe poseer ciertas habilidades emocionales en el entorno educacional, ya que este es un espacio privilegiado de socialización emocional, donde niños, adolescentes y adultos, con necesidades de educación especial, buscan referentes en cuanto a comportamientos, actitudes, emociones y sentimientos (Cabello 
et al., 2009). Por tanto, los trabajadores de educación, quieran o no, cumplen el rol de "educadores emocionales" (Extremera y Fernández- Berrocal, 2009).

La importancia de los hallazgos de este estudio radica en que, de ser replicado y encontrado los mismos resultados por distintos investigadores, servirá de pauta para implementar técnicas de incremento del bienestar personal en funcionarios de educación especial. La elaboración de los programas debería enfatizar las habilidades y competencias personales, emocionales y sociales que se identificaron en este estudio, a saber: claridad, autoconcepto, reparación, control de impulsos, relaciones interpersonales y solución de problemas, ya que éstas inciden en mayor medida en la satisfacción vital, felicidad subjetiva y resiliencia.

Como un ejemplo de lo anterior, se puede señalar que al término de esta investigación, en la etapa de devolución de resultados a las personas que colaboraron con la misma, se solicitó a los autores la realización de un programa de capacitación orientado al personal administrativo, funcionarios académicos y no académicos que trabajan en educación especial en la región Tarapacá.

\section{REFERENCIAS BIBLIOGRÁFICAS}

Atienza, F. L., Pons, D., Balaguer, I. y García-Merita, M. (2000). Propiedades psicométricas de la escala de satisfacción con la vida en adolescentes. Psicothema, vol. 12, n. 2, 314-319.

Bar-on, R. (1997). Bar-On Emotional Quotient Inventory (EQ-i): Technical manual. Toronto: MultiHealth Systems. In Bar-On, R. y Parker, D. (eds.), The handbook of emotional intelligence. San Francisco: Jossey-Bass.

Bonanno, G. A. (2004). Loss, trauma and human resilience. Have we underestimated the human capacity to thrive after extremely aversive events? American Psychologist, vol. 59, n. 1, 20-28.

Caballero, E. L. y Valero, H. (2004). Discapacidad laboral por trastornos mentales en trabajadores docentes. Revista Cubana de Salud y Trabajo, vol. 5, n. 2, 26-30.

Cabello, R., Ruiz-Aranda, D. y Fernández-Berrocal, P. (2009). Docentes emocionalmente inteligentes. Revista Interuniversitaria de Formación del Profesorado, vol. 13, n. 1, 41-49.

Connor, K. y Davidson, J. (2003). Development of a new resilience scale: The Connor-Davidson Resilience Scale (CD-RISC). Depression and Anxiety, vol. 18, 76-82.

Cuadra, A., Fuentes, L., Madueños, D., Bustos, Y. y Veloso, C. (en prensa). Mejorando el clima organizacional, clima de aula, satisfacción vital y laboral con psicología positiva. Artículo aceptado para publicación en revista Fractal.

Cuadra Peralta, A., Lee Maturana, S. y Sossa Roa, P. (2009). Construcción de perfiles psicográficos de profesores de educación básica de escuelas municipales de Arica. Estudios Pedagógicos, vol. $35, n .1,13-26$.

Cuadra-Peralta, A., Veloso-Besio, C., Ibergaray, M. y Rocha, M. (2010). Resultados de la Psicoterapia Positiva en Pacientes con Depresión. Revista Terapia Psicológica, vol. 28, n. 1, 127-134.

Cuadra-Peralta, A., Veloso-Besio, C., Rubio, Y., Reyes, L. y Vilca, J. (2010). Efecto de un programa de psicología positiva e inteligencia emocional sobre satisfacción laboral y vital. Revista Salud y Sociedad, vol. 1, n. 2, 101-112.

Cuadra-Peralta, A., Veloso-Besio, C., Puddu-Gallardo, G. y Salgado-García, P. (en prensa). Impacto de una intervención en psicología positiva sobre síntomas depresivos y satisfacción vital en adultos mayores. Artículo aceptado para publicación en revista Psicología: Reflexión y Crítica.

Diener, D., Emmons, R. A., Larsen, R. J. y Griffin, S. (1985). The Satisfaction with Life Scale. Journal of Personality Assessment, vol. 49, 71-75. 
Diener, E. D., Suh, E., Lucas, R. y Smith, H. (1999). Subjective well-being: three decades of progress. Psychological Bulletin, vol. 125, n. 2, 276-302.

Espinoza, I. y Zúñiga, M. (2003). Estudio Inicial en Chile del EQ-I-BAR-ON EMOTIONAL. Tesis. Arica: Departamento de Filosofía y Psicología, Universidad de Tarapacá.

Extremera, N., Durán, A. y Rey, L. (2005). La inteligencia emocional percibida y su influencia sobre la satisfacción vital, la felicidad subjetiva y el engagement en trabajadores de centros para personas con discapacidad intelectual. Ansiedad y Estrés, vol. 11, n. 1, 63-73.

Extremera, N., Durán, A. y Rey, L. (2010). Recursos personales, síndrome de estar quemado por el trabajo y sintomatología asociada al estrés en docentes de enseñanza primaria y secundaria. Ansiedad y Estrés, vol. 16, n. 1, 47-60.

Extremera, N., Fernández-Berrocal, P. y Durán, A. (2003). Inteligencia emocional y burnout en profesores. Encuentros en Psicología Social, n. 1, 260-265.

Fernández-Berrocal, P. y Extremera, N. (2009). La inteligencia emocional y el estudio de la felicidad. Revista Interuniversitaria de Formación del Profesorado, vol. 66, n. 23,3, 85-108.

Fernández-Berrocal, P., Extremera, N. y Ramos, N. (2004). Validity and reliability of the Spanish modified version of Trait Meta-Mood Scale. Psychological Reports, n. 94, 751-755.

Fernández-Berrocal, P. y Ruiz, D. (2008). La inteligencia emocional en la educación. Education \& Psychology, vol. 6, n. 15, 421-436.

Gabel, R. (2005). Inteligencia emocional: perspectivas y aplicaciones ocupacionales. Documentos de trabajo, 16. Universidad ESAN, Lima. Web: http://www.esan.edu.pe/publicaciones/2009/12/07/ DocTrab16.pdf

García, M. (2002). El bienestar subjetivo. Escritos de Psicología, n. 6, 18-39.

Gil-Monte, P. R. y Peiró, J. M. (1996). Un estudio sobre antecedentes significativos del síndrome de quemarse por el trabajo (burnout) en trabajadores de centros ocupacionales para discapacitados psíquicos. Psicología del Trabajo y de las Organizaciones, vol. 12, n. 1, 67-80.

Hervás, G. (2009). Psicología Positiva: Una introducción. Revista Interuniversitaria de Formación del Profesorado, vol. 66, n. 23,3, 23-41.

Liberalesso, A. (2002). Bienestar subjetivo en la vida adulta y la vejez: hacia una psicología positiva en América Latina. Revista Latinoamericana de Psicología, vol. 34, n. 1-2, 55-74.

Maslach, C. y Jackson, S. (1986). Maslach Burnout Inventory Manual (2nd ed.). Palo Alto, California: Consulting Pschologists.

Mayer, J. D., Roberts, R. D. y Barsade, S. G. (2008). Human abilities: Emotional Intelligence. Annual Review of Psychology, n. 59, 507-536.

Mayer, J. D., Salovey, P. y Caruso, D. (2000). Models of emotional intelligence. En R. J. Sternberg (Ed.), Handbook of Intelligence (2nd ed.) (pp. 396, 420). New York: Cambridge.

Padrós, F., Martínez, M. P., Gutiérrez-Hernández, C. y Medina, M. (2010). La psicología positiva. Una joven disciplina científica que tiene como objeto de estudio un viejo tema, la felicidad. Uaricha, Revista de Psicología, vol. 14, 30-40.

Páez, D., Fernández, I., Campos, M., Zubieta, E. y Casullo, M. (2006). Apego seguro, vínculos parentales, clima familiar e inteligencia emocional: socialización, regulación y bienestar. Ansiedad y Estrés, vol. 12, n. 2-3, 329-341.

Palomera, R. y Brackett, M. (2000). Frequency of positive affect as a possible mediator between perceived emotional intelligence and life satisfaction. Ansiedad y Estrés, vol. 12, n. 2-3, 231-239.

Paula, I. (2005). Estudio de casos sobre el distrés laboral en profesionales de la educación especial. Mapfre Medicina, vol. 16, n. 1, 36-51.

Salovey, P., Mayer, J.D., Goldman, S.L., Turvey, C. y Palfai, T.P. (1995). Emotional attention, clarity and repair: exploring emotional intelligence using the Trait Meta-Mood Scale. In J.W. Pennebaker (ed.), Emotion, disclosure and health (pp. 125-154). Washington: American Psychological Association 
Salovey, P., Rothman, A. J., Detweiler, J. B. y Steward, W. T. (2000). Emotional states and physical health. American Psychologist, vol. 55, n. 1, 110-121.

Seligman, M. y Czikszentmihalyi, M. (2000). Hapiness, excellence, and optimum human function. American Psychologist, vol. 55, 5-183.

Schneider, B. y Bowen, D. (1993). The service organization: human resources management is crucial. Organizational Dynamics, vol. 21, 39-52.

Ugarriza, N. (2001). La evaluación de la inteligencia emocional a través del inventario de BarOn (I-CE) en una muestra de Lima Metropolitana. Persona, n. 4, 129-160.

Yu, X. y Zhang, J. (2007). Factor analysis and psichometric evaluation of the Connor-Davidson Resilience Scale (CD-RISC) with chinese people. Social Behavior and Personality: an International Journal, vol. 35, n. 1, 19-30.

Zamarrón, Mª D.(2006). El bienestar subjetivo en la vejez. Lecciones de Gerontología, n. 52, 4-18. 